\title{
On Computing Probabilities of Dismissal of 10b-5 Securities Class-Action Cases
}

\author{
Sumanta Singha ${ }^{\mathrm{a}, *}$, Steve Hillmer ${ }^{\mathrm{a}}$, Prakash P. Shenoy ${ }^{\mathrm{a}}$ \\ ${ }^{a}$ University of Kansas, School of Business, Lawrence, KS 66045, USA.
}

\begin{abstract}
The main goal of this paper is to propose a probability model for computing probabilities of dismissal of 10b-5 securities class-action cases filed in United States Federal district courts. By dismissal, we mean dismissal with prejudice in response to the motion to dismiss filed by the defendants, and not eventual dismissal after the discovery process. The proposed probability model is a hybrid of two widely-used methods: logistic regression, and naïve Bayes. Using a dataset of 925 10b-5 securities class-action cases filed between 2002 and 2010, we show that the proposed hybrid model has the potential of computing better probabilities than either LR or NB models. By better, we mean lower root mean square errors of probabilities of dismissal. The proposed hybrid model uses the following features: allegations of generally accepted accounting principles violations, allegations of lack of internal control, bankruptcy filing during the class period, allegations of Section 11 violations of Securities Act of 1933, and short-term drop in stock price. Our model is useful for those insurance companies which underwrite Directors and Officers liability policy.
\end{abstract}

Keywords: probability, logistic regression, naïve Bayes, hybrid model, 10b-5 security class-action cases

\section{Introduction}

Decision support system (DSS) tools have been an integral part of effective decision making. Over the years, DSS tools have enriched managerial judgement by turning data-driven insights

\footnotetext{
${ }^{*}$ Corresponding author

Email addresses: sumanta.singha@ku.edu (Sumanta Singha), hillmer@ku.edu (Steve Hillmer), pshenoy@ku.edu (Prakash P. Shenoy)

Preprint submitted to Journal of Decision Support Systems

April 25, 2017
} 
into actionable solutions. Much of recent developments in decision making is in model-based 5 management support which incorporates knowledge and models for judgement and decision $[1,2]$. An example of such system includes credit approval applications that access credit scores and other relevant information to approve/deny credit card applications. Model-based DSS finds applications in wide variety of domains such as quality management [3], energy planning [4], project portfolio selection [5], and others. In this paper, we aim to develop a model-based DSS for the D\&O insurance companies in the context of 10b-5 securities class-action lawsuits.

Securities class-actions are lawsuits filed by shareholders due to violation of securities laws. Some common class-action allegations are fraudulent disclosure, misleading forecast, violation of securities laws, insider trading and financial restatements. It is well known that securities classactions can inflict serious damage to defendant's financial health and stock market performance $[6,7]$. In recent years, nearly $35-40 \%$ of all class-action suits allege security frauds and account for approximately $75 \%$ of all settlement awards $[8,9]$. Since 1996, over 4,100 security class-actions have been filed and approximately $\$ 87$ billion has been dispensed in settlements ${ }^{1}$. As a result, virtually every public corporations in U.S. buy Directors and Officers (D\&O) coverage to safeguard its directors and officers from class-action liabilities [10, 11].

In this context, the role of $\mathrm{D} \& \mathrm{O}$ underwriters and risk managers becomes crucial. While past literatures $[10,12]$ provide some guidance on preemptive risk management (e.g. predicting corporate governance risk), the reactive risk management aspects have not been well studied. In particular, it is still an open question for the insurance companies to decide whether to 'settle' or 'fight' (on behalf of the defendant) when facing a class-action litigation. Given the high cost 25 of penalties, clearly, an early settlement can potentially benefit the insurance company when the probability of dismissal is low i.e. the lawsuit is likely to be ruled in favor of the plaintiff. This explains why insurance companies may be interested in predicting probability of dismissal early in the trial. To answer this research question, we propose a predictive model that can estimate probability of dismissal at an early stage, solely based on the allegations made by a plaintiff. This paper investigates how an enabling decision support system can lead to improved decision-making

\footnotetext{
${ }^{1}\langle$ http://securities.stanford.edu/ $\rangle$
} 
for the insurance companies.

There are several challenges that must be addressed while attempting to build a probability model. The proposed model must be reliable, well-calibrated, efficient, and sensitive to error. In order to simultaneously achieve these multiple objectives, we adopt a multi-pronged strategy, i.e., Markov blankets for feature set selection; root mean square errors (RMSE) as a model evaluation metric; reliability diagram for model calibration; and non-parametric bootstrapping for computing standard deviation of test set errors. We also show that our model produces lower error compared to a recent method [13]. We discuss in further details in Section 8. Note that this paper is about building a decision tool for predicting probability of dismissal in securities class-action cases based on a dataset of 925 instances. We find that a hybrid of two widely-used methods: logistic regression (LR), and naïve Bayes (NB) performs better than pure LR and pure NB for this dataset. The strengths of our model are that it can simultaneously incorporate continuous features and features with missing features, is efficient and yet simple to use.

Two natural questions to ask at this juncture. First, why use a hybrid model? Second, when does a hybrid model perform better than the constituent methods?. Below we answer these two questions together. Referring to the "no free lunch" theorem in [14], we state that no single computational view solves all the problems, since each one has its own domain of competence. Hybrid models have been studied extensively in the past with respect to classification $[15,16]$. It is well known that hybrid methods seek to exploit the strengths of the individual components, 50 obtaining enhanced performance for the combination [17-19]. [20] provides an analytical proof that hybrid classifier works better under some conditions. In the machine learning domain also, different hybrid models combining generative and discriminative approaches have been proposed, such as hybrid generative-discriminative models [21], mixed log-likelihood model [22, 23], multi-conditional learning models [24], Bayesian trade-off [25], H-bayes model [26], and JoDiG approach [27]. [28] compare logistic regression and nave Bayes models. Their conclusions are that asymptotically LR models do better than NB, but that NB models reach their asymptotic accuracy faster than LR models. [29] observe that the generative approach enjoys better classification performance than its discriminative counterpart when the model is correctly specified. However, when the 
model is misspecified, the performance of discriminative model improves as the training set size increases. Between these two extremes, there is a region where hybrid model may outperform both generative and discriminative techniques. This, once again, substantiates the celebrated " $n$ o free lunch" theorem in [14] and answers our second question. To explore whether hybrid method is always optimal for all domains is beyond the scope of this paper.

Our sole objective is to propose a model that enables D\&O insurers to identify which features contribute to the dismissal (or settlement) of a class-action and compute the probability of dismissal conditional on their realizations. Our model not only helps the D\&O insurers to make an early assessment of risk, but also acts as an useful tool for the future. The study also provides useful insights to the Securities and Exchange Commission (SEC), other policymakers, and academicians who study policy implications and implement judicial reforms.

The remainder of the paper is organized as follows. The next section contains a review of related works on class-action lawsuits. In Section 3, we summarize the contributions of this paper. Section 4 describes the dataset and explains the complexities involved in data preparation. In the subsequent three sections, we sketch the basics of NB, LR and hybrid method, their assumptions, strengths and weaknesses. In Section 8, we describe the feature selection methodology and their nuances. In Section 9, we present our results and compare the models. Finally, in Section 11, we summarize our findings and conclude.

\section{Related Work}

Existing literatures on securities class-actions are broadly of two types. The first category is comprised of those which examine the effect of the U.S. Private Securities Litigation Reforms Act (PSLRA) of 1995 on class-action outcomes and is extremely rich in content. Motivated by PSLRA, these papers explore which factors contribute to the dismissal (or settlement) of a classaction and influence the settlement size conditional on settlement. The significant contributions in this category are by $[10,11,30-32]$. We summarize some of their contributions below.

In the post-PSLRA period, [32] finds that the allegation of financial restatements makes a case more likely to be settled, while the allegations of insider trading and misleading forecast are 
insignificant for the outcomes. [30,31] study the lead plaintiff provision of PSLRA and examine whether institutional lead plaintiff increases the settlement size. Their findings suggest that though institutional lead plaintiff increases the average settlement, the lead plaintiff provision of PSLRA is generally ineffective. It is learnt that (i) provable loss, (ii) total asset of the defendant firm, and (iii) presence of an SEC enforcement action, all positively influence the settlement amount. [33] investigates the kind of cases that are ultimately dismissed/settled and find that security classaction cases that allege financial restatements and SEC enforcement actions are less likely to be dismissed.

In contrast, the second category is relatively nascent and contains very few literatures, which focus on predicting the probability of dismissal/settlement in a class-action case. Unlike the first category, here we deal with probability models that can predict probabilities. The significant contributions in this category are by [34] and [13] . Using a dataset of 155 instances, [34] employs a logistic regression model to determine which allegations are correlated with the outcome of the motion to dismiss and by inference, whether these allegations influence the decisions of the ruling judges. They find that violation of generally accepted accounting principles (GAAP) and allegation of false forward looking guidance favour settlement, while insider trading allegation may or may not be significant (at $5 \%$ significance level) and the courts differ in their views. The significant features are selected based on the $p$-value of the regression coefficients. Though their model is essentially a classification model, [34] only focuses on determining the significant features that are correlated with the outcome and hence does not provide any model performance measure. As a result, we only qualitatively compare our results with [34].

We now turn to the work by [13], which is most relevant in our context. Using a dataset of nearly 1200 instances and 18 features primarily from RiskMetrics, ${ }^{2}$ they propose a Bayesian hierarchical composite model that estimates (i) the probability of dismissal, and (ii) the settlement size conditional on non-dismissal. The portion of the model for computing probability of dismissal is a logistic regression model. Some of the 18 features they use are relevant for computing probability of dismissal, and some for predicting settlement size, and it is not clear which features are relevant

\footnotetext{
${ }^{2}\langle$ https : //www.issgovernance.com/governance-solutions/securities-class-action-services/〉
} 
for each task.

In this paper, we develop a probability model by using a recent technique, called a hybrid model,

that combines logistic regression (LR), and naïve Bayes (NB) in one model. Using a dataset of 925 instances of 10b-5 securities class-action cases filed in U.S. Federal district courts during $2002-$ $2010,^{3}$ we compute the probability of dismissal based on a set of features. Rule 10b-5 violation deals with securities fraud and appears in approximately $82 \%$ of all security class-actions filed in last 5 years [35].

Using a new algorithm for the construction of a hybrid model, we find that only 5 features are significant for computing the probabilities of class-action outcomes. They are: (i) GAAP violation, (ii) lack of internal control, (iii) whether the defendant has filed for bankruptcy in the class period, (iv) violations of Section 11 of Securities Act of 1933; and (v) percentage of sudden short-term drop in share price alleged in the consolidated complaint. A predictive model is proposed that determines the probability of dismissal based on these features. In the next section, we contrast our work with [13] and show that our model provides higher accuracy, in addition to being tolerant to missing data.

\section{Contribution}

Our work contributes to the existing literature on class-action cases in the following ways.

1. First, we define the notion of 'dismissal' from the viewpoint of D\&O insurance company, which is virtually always the principal party at interest. In our model, by 'dismissal' (the class variable), we mean initial dismissal in response to the motion to dismiss filed by the defendants, and not the eventual dismissal that the earlier works have studied. This new definition also complements the underlying spirit of PSLRA, which lays emphasis on heightened pleading requirements and disallows the plaintiff from obtaining discovery prior to disposal of defendant's motion to dismiss. The idea is to eliminate frivolous filings that do not survive the test of motion to dismiss. Clearly, defendant's motion to dismiss in post-PSLRA

\footnotetext{
${ }^{3}\langle$ http://securities.stanford.edu/ $\rangle$
} 


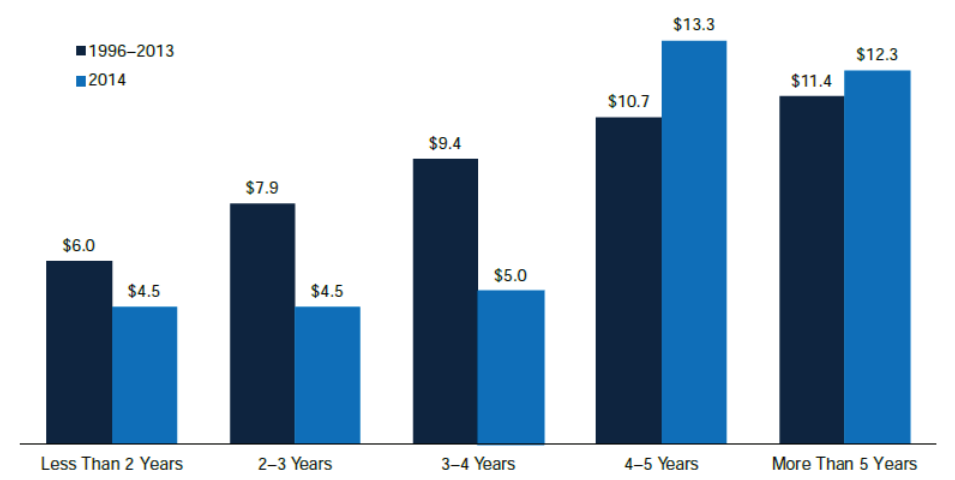

Figure 1: Median settlement (million $\$$ ) by duration from filing date to settlement date (1996-2014)

regime becomes a milestone and in that light, our definition of initial dismissal appears more appealing. Once the motion to dismiss is denied, the next phase is discovery, which is very expensive and all expenses including settlement amount are paid generally by the insurance company under the $\mathrm{D} \& \mathrm{O}$ coverage. So even if the case is eventually dismissed, the insurance company is saddled by huge expenses. Hence, it is prudent for the D\&O company to assess the probability of dismissal before motion to dismiss is disposed of and settle the case sooner if necessary, since settlement amount increases with time (see Figure 1).

2. Next, in contrast to earlier works (see [13]), which use logistic regression, we propose to use a hybrid model. Hybrid model has some advantages over pure LR and pure NB models, and is a relatively new technique. Using the dataset on 10b-5 class-action cases, we show that this approach in the context of this problem has better predictive accuracy than pure LR and pure NB models. [13] uses a graphical technique (reliability diagram) for model evaluation and conclude that their 'settlement/dismissal model' is well calibrated, though the model errors are not reported. For the purpose of comparison, we estimate the RMSE from the reliability diagram, which are approximately $6.01 \%$ for the training set and $11.17 \%$ for the test set. We show that our hybrid model has a training set RMSE of $4.12 \%$ and a test set RMSE of $9.30 \%$, an improvement over the model by [13].

3. Also, our hybrid model differs from those suggested in the literature [26, 27]. [26] uses LR as a discriminative component, and NB as a generative component. All features are used in the hybrid model. They start with all features in the NB part, and then move one feature at a 
time to the LR part, in a greedy fashion, as long as the classification error decreases, and stop otherwise. [27] also uses LR as the discriminative component, but use [36] linear discriminant

\section{Data}

In this section, we describe our dataset and explain the process of labeling each instance, in the light of our new notion of 'dismissal'. The sample is comprised of 925 instances of securities classaction cases filed in various U.S. Federal district courts between 2002-2010. Why 2002-2010? The Sarbanes-Oxley Act of 2002 (often shortened to SOX) is legislation passed by the U.S. Congress to protect shareholders and the general public from accounting errors and fraudulent practices in the enterprise. Most of the class-action cases in 2011 onwards are still pending resolution. Our primary source of data is Stanford Securities Class Action Clearinghouse, ${ }^{4}$ which keeps track of all Federal

\footnotetext{
${ }^{4}\langle$ http://securities.stanford.edu/ $\rangle$
} 
securities class-action cases since the passage of PSLRA. SCAC provides historical information

of securities class action cases with accompanying full-text complaints, motions, dockets, judicial opinions and other major court filings. The data collection was carried out by select graduate law students at University of Kansas, over a period of 6 months. Engaging law students is intended to lend credibility to our dataset from legal viewpoint; making sure that the dataset is unbiased and comprehensive. The dataset is further cross-checked with a commercial dataset (Advisen's Master Significant Cases \& Actions Database ${ }^{5}$ ) for accuracy, and in case of disagreements, we verify by reading the consolidated complaints whether we or Advisen's database have the correct information.

Data collection involves two primary tasks; first, to select instances for our dataset and second, to identify the relevant features that we use to build the model. We independently select 970 class-action cases filed between 2002 - 2010, for which the outcomes to 'motion to dismiss' are known. Our selection is not subject to any pre-determined bias with regard to industry sector, type of plaintiff or any other attribute. Next, we select the relevant features for our model. A total of 19 features are shortlisted, that were commonly alleged in various consolidated complaints. Our assumption is that outcomes are merit-based and depend on the strength of the allegations in consolidated complaint. This assumption is reasonable and consistent with the philosophy of PSLRA. In a pilot study carried out by [39], only 6 of these 19 features are found relevant for predicting dismissal. Subsequently, we further add 3 additional features (last 3 in the list below). These 9 features are:

1. $G P=$ whether GAAP violations is alleged (1) or not $(0)$;

2. $I I=$ whether lead plaintiff is an institutional investor (1) or not (0);

3. $B R=$ whether the defendant has filed for bankruptcy in the class period (1) or not (0);

4. $S 11=$ whether violations under Section 11 of Securities Act 1933 is alleged (1) or not (0);

5. $R F=$ whether company financials are restated during the class period ${ }^{6}(1)$ or not $(0)$; and

6. $S T D=$ the maximum $\%$ short-term (1 to 5 working days) drop in share price alleged;

\footnotetext{
${ }^{5}$ /http://www .advisenltd.com/2015/02/27/mscad-methodology-no-two-databases-are-the-same/ $\rangle$

${ }^{6} \mathrm{~A}$ 'class period' is a specific period of time in which the unlawful conduct is alleged to have occurred.
} 
7. $S I=$ whether investigation is initiated against the defendant by SEC (1) or not (0);

8. $I C=$ whether the complaint alleges lack of internal control (1) or not (0);

9. $I S=$ whether the complaint alleges insider selling (1) or not (0);

Except $S T D$, which is a continuous feature, all other features have binary outcomes, true (1) or false (0), as reported in consolidated complaint. In this problem, the class variable $(C)$ is a to dismiss' filed by the defendants. In these cases, the plaintiffs are not entitled to refile another lawsuit for the same claim. These cases were classified as dismissed. In another 211 cases, the ruling judge dismissed the cases without prejudice, which entitles the plaintiff the right for re-filing another suit later on the same claim. This begins the entire process once again and follows the

\footnotetext{
${ }^{7}$ In the literature, there exist instances of 'strike suits' [31], in which the ratio of settlement to 'provable loss' is extremely low, often indicating the frivolousness of allegations. In such cases, settlement acts more as an exit route and does not imply submission of guilt. We did not engage in such subtleties
} 
same course of events. For the sake of brevity, we only summarize the results. 125 of these 211 cases were classified as dismissed and remaining 86 cases as not dismissed.

\section{Type 2 : Mutually settled prior to ruling on motion to dismiss}

There are 176 cases in which defendants and plaintiffs agreed to settle the case before 'motion to dismiss' was ruled by the judge. Such cases were classified as not dismissed indicating possible merits in the case in favor of the plaintiff.

\section{Type 3: Voluntarily withdrawn by the plaintiffs}

19 such cases were voluntarily withdrawn by the plaintiffs before the court's ruling of the 'motion to dismiss', and hence they were classified as dismissed.

\section{Type 4: Motion to dismiss denied}

The court had rejected the defendant's 'motion to dismiss' and proceeded to discovery in 249 instances. Only 5 of these cases were eventually dismissed by the court (after a period of 3-5 years of discovery), and remaining cases were settled by the defendant. In the light of our new definition of dismissal, all 249 instances were classified as not dismissed.

In summary, the class outcomes are as follows : \# dismissed $=414$, and $\#$ not dismissed $250=511$. In Figure 2, we present the descriptive statistics of the 9 features across each class outcome dismissed and not dismissed. Short term drop (STD) is a continuous feature, but the depiction in Figure 2 is a discretized version where $S T D=1$ means $S T D>42.2 \%$, and $S T D=0$ means $S T D \leq 42.2 \%$ (discretization of $S T D$ is described in greater detail in Section 8). There are no instances of missing data. Finally, the dataset is randomly partitioned into a training set (90\%) and a test set (10\%). As a result, the training set contains 832 cases and test set contains remaining 93 cases.

\section{Naïve Bayes}

A naïve Bayes (NB) model is a Bayesian classifier that assumes mutual conditional independence of the features given the class variable (see Figure 3). This assumption reduces the complexity of

the model (\# parameters), which makes it very robust. Suppose that class variable $C$ has two outcomes (e.g., dismissed $(d)$ and not dismissed $(n d))$ and the Markov blanket of $C$ has $n$ features 


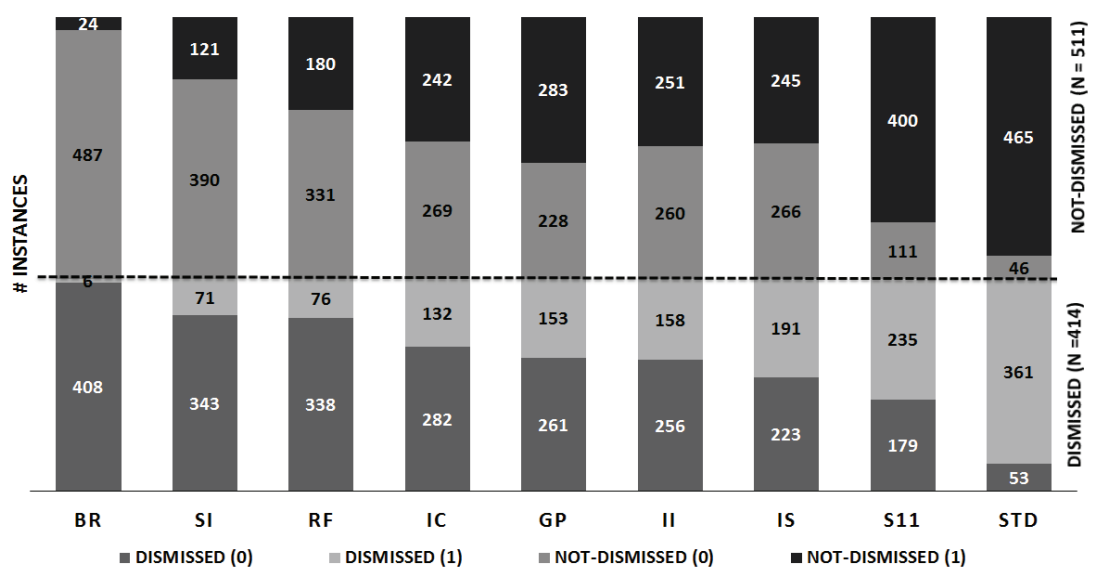

Figure 2: Descriptive statistics of all features in the dataset.

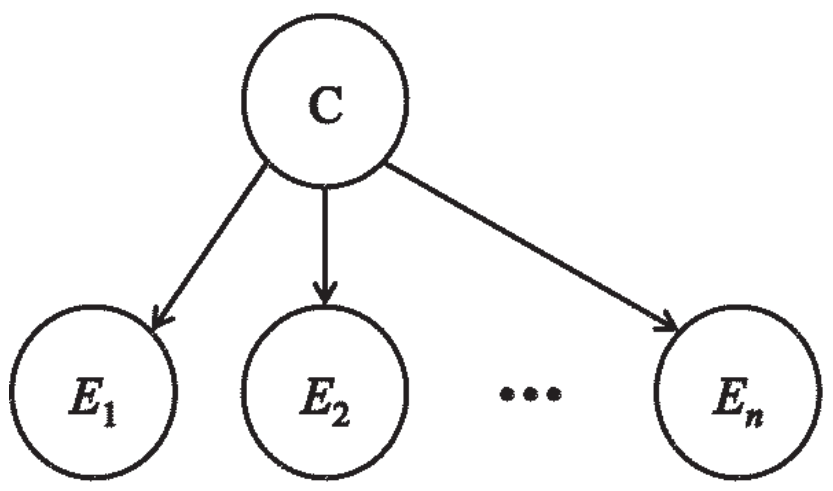

Figure 3: A Naïve Bayes Model

$\mathbf{E}=\left(E_{1}, \ldots, E_{n}\right)$. The assumption of conditional independence means that the joint probability distribution factors as follows:

$$
P(C, \mathbf{E})=P(C) \prod_{i=1}^{n} P\left(E_{i} \mid C\right)
$$

A NB classifier first learns the joint probability $P(C=d, \mathbf{E})$ and then use Bayes' rule to compute the posterior probability distribution $P(C=d \mid \mathbf{e})$ for every new instance $\mathbf{e}=\left(e_{1}, e_{2}, \ldots, e_{n}\right)$ that we wish to classify. Thus,

$$
P(C=d \mid \mathbf{e})=\frac{P(C=d) \prod_{i=1}^{n} P\left(e_{i} \mid C=d\right)}{P(\mathbf{e})}
$$




$$
P(C=n d \mid \mathbf{e})=\frac{P(C=n d) \prod_{i=1}^{n} P\left(e_{i} \mid C=n d\right)}{P(\mathbf{e})}
$$

Dividing Eq. (2) by Eq. (3), we get;

$$
O(C=d \mid \mathbf{e})=O(C=d) \prod_{i=1}^{n} L\left(C=d, e_{i}\right)
$$

where $O(C=d \mid \mathbf{e})$ denotes the posterior odds $\frac{P(C=d \mid \mathbf{e})}{P(C=n d \mid \mathbf{e})}$ for dismissed, $O(C=d)$ denotes the prior odds $\frac{P(C=d)}{P(C=n d)}$ for dismissed, and $L\left(C=d, e_{i}\right)$ denotes the likelihood ratio $\frac{P\left(e_{i} \mid C=d\right)}{P\left(e_{i} \mid C=n d\right)}$ for dismissed from observed value $e_{i}$ of feature $E_{i}$. Once we have computed posterior odds for $C=d$, we can compute the posterior probability $P(C=d \mid \mathbf{e})$ as follows:

$$
P(C=d \mid \mathbf{e})=\frac{O(C=d \mid \mathbf{e})}{O(C=d \mid \mathbf{e})+1}
$$

Eqs. (4) and (5) enable us to compute the posterior probability for dismissed using the NB classifier given observed attribute values e. For a binary class variable $C$ and $n$ binary features, we have $2 n+1$ parameters. If a feature has a missing (or unobserved) value, its corresponding likelihood ratio is 1 and we can disregard such features from Eq. (4). So NB is tolerant to missing data.

The conditional independence assumptions of a NB classifier are often not satisfied in a real dataset. However, the model classification performance may still be good in practice although the probabilities may not be well calibrated [40]. Presence of continuous features having non-gaussian distribution imposes some problems. One common solution is to discretize the continuous features into a finite number of bins, though such discretization may result in loss of information. Also, the number of parameters increases with the \# bins. For each feature with $k$ bins, we have $c *(k-1)$ parameters, where $c$ is the number of classes. We discuss discretization in more detail in Section 8 . 


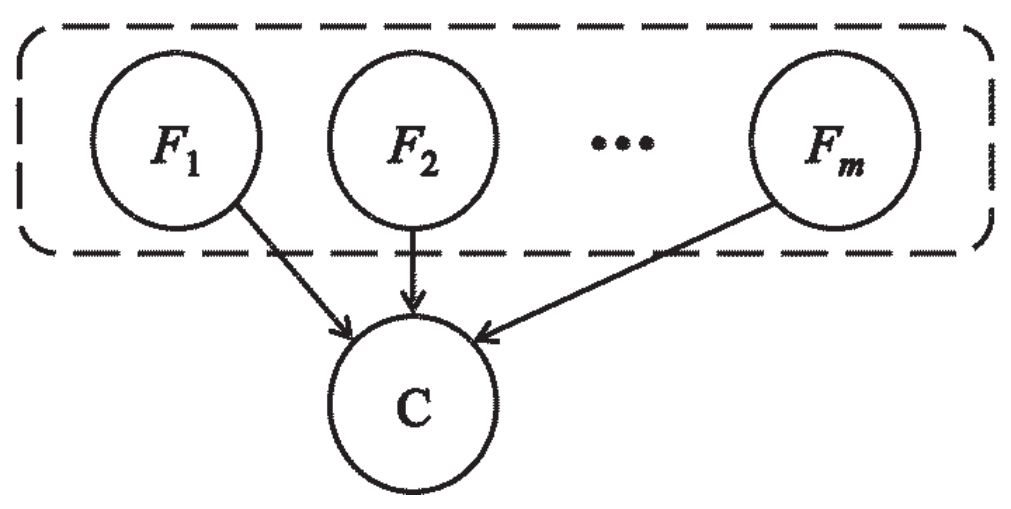

Figure 4: A Logistic Regression Model

\section{Logistic Regression}

Logistic regression (LR) is a conditional probability model that estimates the conditional probability directly from the data. Unlike NB, a logistic regression model makes no assumptions about conditional independence of the features. It only assumes that the posterior log odds of the class variable is a linear function of the features. LR model can handle both discrete or continuous real-valued features, but is not tolerant to missing data. If we have a categorical feature with $k$ nominal values, we have to represent such a feature with $k-1$ boolean features with values in $\{0,1\}$.

Figure 4 shows a logistic regression model as a Bayesian network. The dotted oval containing the feature variables denotes that the graphical structure of the feature variables is unspecified. Given observed values of all feature variables, the said graphical structure is irrelevant to the posterior marginal of the class variable.

Given a set of $m$ discrete or continuous features $\mathbf{F}=\left(F_{1}, \ldots, F_{m}\right)$, and corresponding observation vector $\mathbf{f}=\left(f_{1} \ldots, f_{m}\right)$, we have:

$$
\ln (O(C=d \mid \mathbf{f}))=\beta_{0}+\beta_{1} f_{1}+\beta_{2} f_{2}+\ldots+\beta_{m} f_{m}
$$

By taking the anti-logarithm of both sides of Eq. (6), we get:

$$
O(C=d \mid \mathbf{f})=e^{\beta_{0}+\beta_{1} f_{1}+\ldots+\beta_{m} f_{m}}
$$


We compute the posterior probability $P(C=d \mid \mathbf{f})$ as follows:

$$
P(C=d \mid \mathbf{f})=\frac{O(C=d \mid \mathbf{f})}{O(C=d \mid \mathbf{f})+1}
$$

Eq. (8) enables us to compute the posterior probability for dismissed using the LR classifier independence allows us to compute the conditional $P(C \mid \mathbf{f})$ in the LR part using standard toolkits 


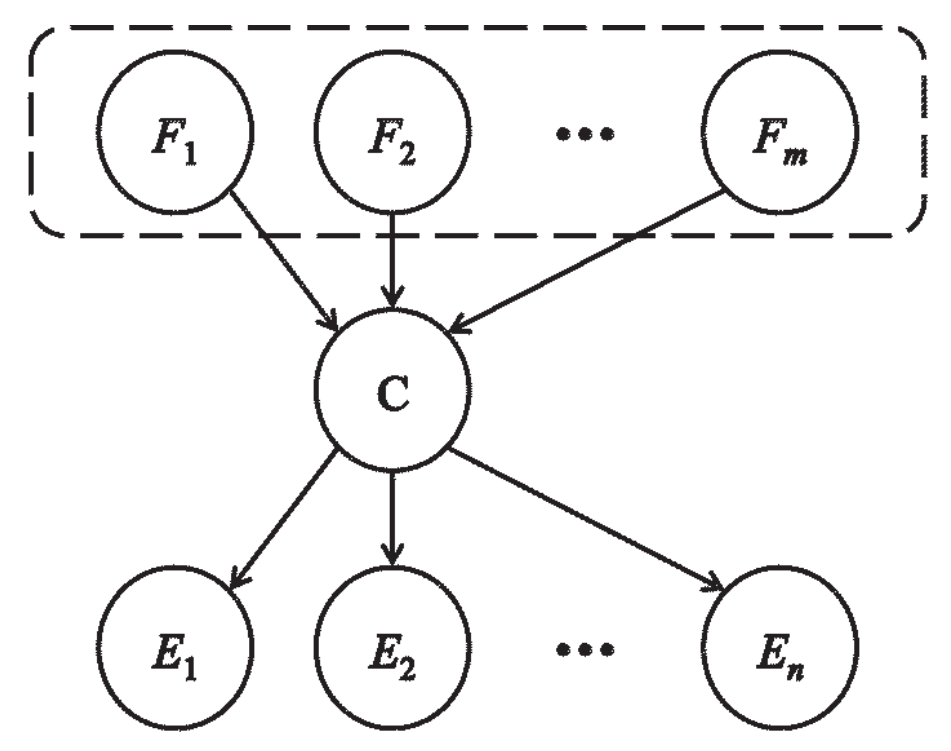

Figure 5: A Hybrid LR-NB Model

for logistic regression, while the conditionals $P\left(e_{j} \mid C\right)$ for each value $e_{j}$ of $E_{j}$, can be learnt using the standard methods for naïve Bayes.

Making inference in a hybrid model is simple. For the simplicity of exposition, assume $C$ is a binary variable, as before. Using variable elimination [42], if we eliminate the features in the LR part of the model, we get the posterior odds $O(C=d \mid \mathbf{f})$ as given by Eq. (7). If we now eliminate the features in the NB part of the model, we get the posterior of $C$ as given by Eq. (4), but with $O(C=d \mid \mathbf{f})$ replacing the prior odd $O(C=d)$. Formally, if $\mathbf{f}=\left(f_{1}, \ldots, f_{m}\right)$ and $\mathbf{e}=\left(e_{1}, \ldots, e_{n}\right)$, then:

$$
O(C=d \mid \mathbf{f}, \mathbf{e})=e^{\beta_{0}+\sum_{i=1}^{m} \beta_{i} f_{i}} \prod_{j=1}^{n} L\left(C=d, e_{j}\right)
$$

Eq. (9) is the main equation for making exact inference from a hybrid model. If all features in the NB part of the model and the class variable are binary, then the number of parameters in a hybrid model is $m+1+2 n$. Thus, a hybrid model retains the simplicity of LR and NB models. 


\section{Feature Selection and Construction of a Hybrid Model}

In this section, we describe how we select features for a hybrid model, and provide an algorithm for the construction of hybrid model. Our method is implemented for the the 10b-5 security classaction dataset.

Step 1: Markov Blanket Estimation. In the first step, we estimate a Markov blanket of the class variable $C$. By definition, a node is conditionally independent of all other nodes given its Markov blanket. For example, In a Bayesian network, a variable's Markov blanket includes its parents, its children, and co-parents of its children. Thus, the knowledge of the features in the Markov blanket of class $C$ alone is sufficient to predict the class outcome. We use the training set to estimate the Markov blanket. The Markov blanket of class variable $C$ is estimated using learn.mb command in bnlearn package in $\mathrm{R}[43]$.

Various constraint-based algorithms [44] exist in literature that use conditional independence tests to estimate the Markov blanket. These algorithms compute the Markov blanket directly, without first estimating a graphical model. We use 4 different constraint-based algorithms: (i) grow shrink [45]; (ii) incremental association [46]; (iii) fast incremental association [47]; and (iv) interleaved incremental association [46], and 10 different conditional independence tests ${ }^{8}$ as implemented in bnlearn package for each of these algorithms, resulting in 40 estimates of Markov blanket. We take the union of features in all 40 estimated Markov blankets as the estimated Markov blanket of $C$. The logic is that a feature is excluded if all estimated 40 Markov blankets are unanimous on the exclusion of the feature. This is only one step in feature selection.

The test results show that the estimated Markov blanket of class $C$ contains 6 features: (i) $G P$, (ii) $R F$, (iii) $I C$, (iv) $S 11$, (v) $B R$, and (vi) $S T D$. Thus, given the values of these six features, the remaining three features, $S I, I I$, and $I S$, are irrelevant to the class variable, subject to estimation errors. As we have no missing values in our dataset, we proceed with these 6 features for subsequent model selection and validation exercise.

\footnotetext{
${ }^{8}$ These conditional independence tests are for discrete variables and include asymptotic chi-square tests based on mutual information with and without adjusted degrees of freedom, Monte Carlo permutation test, the sequential Monte Carlo permutation test, the semi-parametric test, shrinkage estimator for the mutual information, classic Pearson's chi-square test for contingency tables with and without adjusted degrees of freedom, the Monte Carlo version of the chi-square test, and the semi-parametric version of the chi-square test.
} 


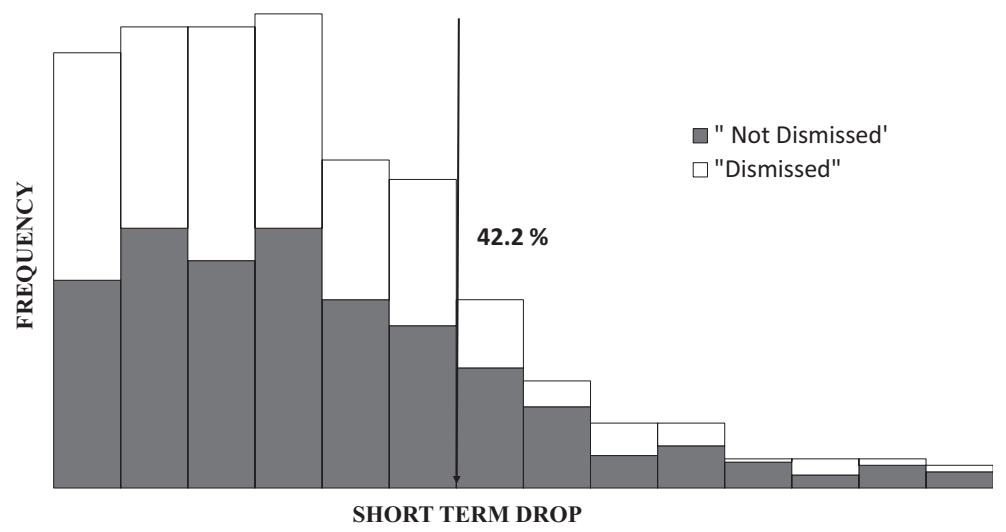

Figure 6: Discretization of Short Term Drop

Step 2: Discretization of Continuous Features. Discretization of a continuous feature is needed only when it is used in the NB part of the hybrid model. [48] presents an extensive survey of various discretization techniques. In particular, multi-level discretization technique by [49] and entropy and MDL based discretization techniques by [50] are very useful. In this paper, we discretize STD into two bins with cutoff value of $42.2 \%$ (i.e., $S T D=0$ if drop is $\leq 42.2 \%$ and 1 otherwise) . This cut-off percentage is arrived at by supervised discretization. One effective way to discretize a continuous feature is to look for specific breakpoints at which the class outcomes are sensitive to the changes in the value of a numeric feature. This implies the proportion of success to failure (likelihood ratio) changes below and above this breakpoint. However, it is not necessary always for such a distinct breakpoint to exist. In such cases, other discretization techniques will work. In our dataset, we observe that ratio of frequency of dismissed to not dismissed exhibits a change around $42.2 \%$ (see Figure 6 ).

Step 3: Searching for Best LR and Best NB Models. With 6 features in hand from Step 1, our goal is to find the best LR and best NB models. By best, we mean lowest RMSE using cross validation. If the number of features is small, we can do a complete enumeration, which involves searching among $2^{q_{1}}-1$ models, where $q_{1}$ is the number of features remaining after step 1 . If the number of 

random splits of the training data. Finally, we compute the average RMSE and the standard error 
Table 1: A Typical example of computation of bin probability and RMSE

\begin{tabular}{|lccc|}
\hline & Avg. predicted prob. & Actual prob. & Sq. error \\
\hline Bin 1 & 0.270 & 0.288 & 0.0003 \\
Bin 2 & 0.320 & 0.355 & 0.0012 \\
Bin 3 & 0.340 & 0.336 & 0.0000 \\
Bin 4 & 0.385 & 0.365 & 0.0004 \\
Bin 5 & 0.438 & 0.442 & 0.0000 \\
Bin 6 & 0.482 & 0.432 & 0.0024 \\
Bin 7 & 0.598 & 0.644 & 0.0021 \\
Bin 8 & 0.654 & 0.625 & 0.0008 \\
Sum of squared errors & & & 0.0072 \\
RMSE & & & 0.0300 \\
\hline
\end{tabular}

of the average RMSE. We do this for all models, and choose a model having the lowest error. A typical example of computation of predicted and true average probability and RMSE is presented in Table 1, and is shown in Figure 7.

Bins-Bias Trade-off. The choice of \# bins (say $k, k=8$ in our case) is critical for model selection. It may be seen that the choice of $k$ not only affects the magnitude of the RMSE of each model, but also affects their ranking. To explain this phenomenon, we have examined RMSE of all 63 LR models with 8-fold CV and two different bin sizes: $k=4$ (see Figure 8) and $k=8$ (see Figure 9). The results show that choice of $k$ gives rise to systemic bias towards selecting models with fewer features when $k=4$, and models with more features when $k=8$, as can be seen from Figures 8 and 9. In these two figures, the first 6 models have 1 feature, the next 13 have two features, etc. For the case of 4-bins (Figure 8), the smallest RMSE is achieved by a model with 2 features (model \#7), whereas for the case of 8-bins (Figure 9), the smallest RMSE is achieved by a model with 5 features (model \#58). The dotted line in Figure 8 and Figure 9 represents \# features in the model.

To explain why this bias occurs, if $k$ denotes the number of bins of the training set, the true probability of dismissal of the $k$ bins can be characterized by $(k-1)$ parameters (assuming that the \# dismissed cases in the training set is known). When we search for models to estimate the true probabilities of dismissal of $k$ bins by minimizing RMSE, assuming that each binary feature 


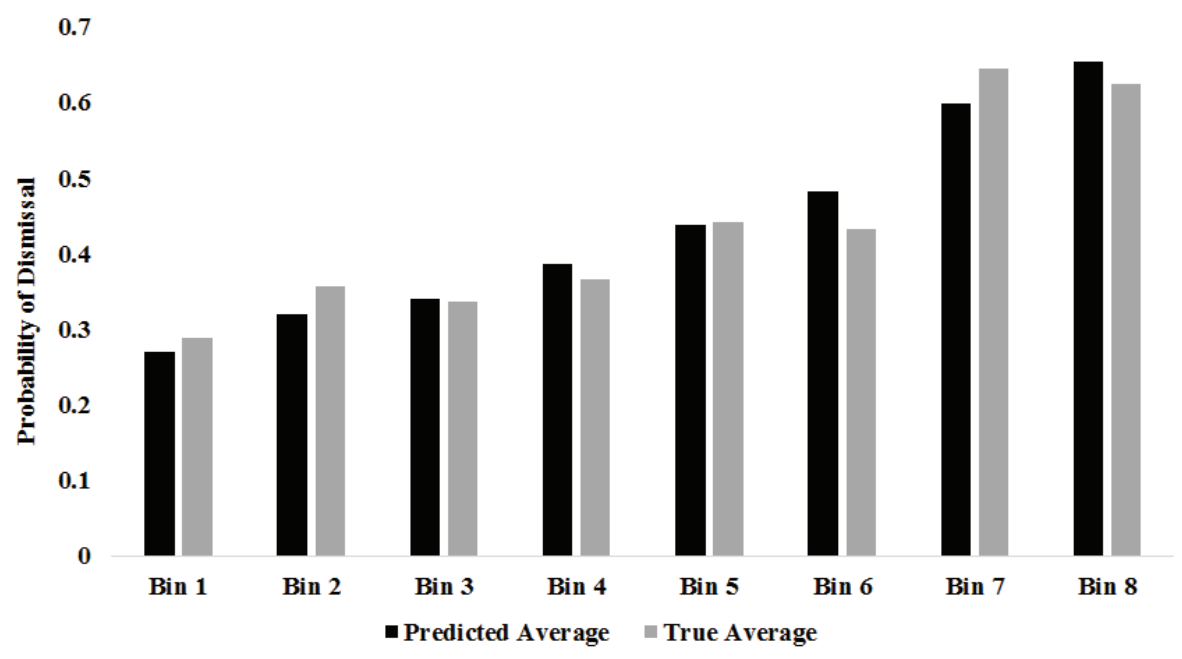

Figure 7: Demonstration of computation of RMSE with 8 bins

accounts for learning at most one of the $(k-1)$ parameters, the process will inherently favor models that have $(k-1)$ or fewer binary features. In contrast, models having more than $(k-1)$ features are unlikely to be chosen though such models may have good predictive power. For example, if $k=1$, we don't need any features to estimate the probability of dismissal for 832 cases. For our example, we split the training set into 8 bins, which allows selection of any model up to 7 parameters in an unconstrained manner. As we have only 6 features in the Markov blanket, the choice of 8 bins is justified.

Step 5: Construction of a Hybrid Model. In this step, we construct a hybrid model. We have 6 features in the Markov blanket and this leads to $\left(3^{6}-1\right)-\left(2^{6}-1\right)-\left(2^{6}-1\right)=602$ possible hybrid models excluding pure LR and pure NB models. If we have a large number of features in the estimated Markov blanket, searching for the best hybrid model is computationally intractable. We propose a heuristic as follows. First, we search for the best LR and best NB models. Next, we search for the best hybrid models from the set of features that are either in the best LR model or in the best NB model, i.e., we assume that if a feature is neither in best LR nor in best NB models, then it will not be in the best hybrid model. This represents our second step in feature selection for constructing a hybrid model.

In the case of our dataset, based on lowest RMSE, the best LR model contains 5 features: (i) 


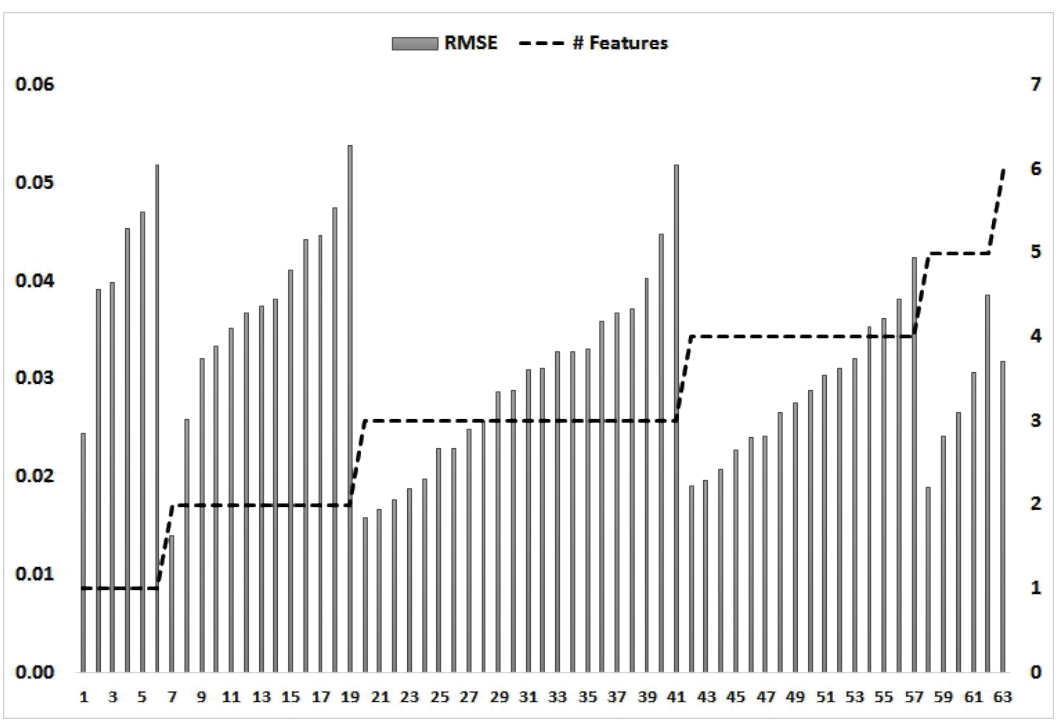

Figure 8: RMSE of LR models with 8-fold CV and 4 bins using the training set

$G P$, (ii) $I C$, (iii) $S T D$, (iv) $B R$, and (v) $S 11$, and the best NB model contains 2 features: (i) $G P$, and (ii) STD. Hence, there are only 5 features that are either in best LR or in the best NB models. Therefore, we need to search only $\left(3^{5}-1\right)-\left(2^{5}-1\right)-\left(2^{5}-1\right)=180$ models to find the best hybrid model (as opposed to 602 models without the heuristic).

Next, we further assume that the best hybrid model must contain all 5 features that are either in best LR or best NB models, and this further reduces our search space. Now we actually need to search only $\left(2^{5}-2\right)=30$ (out of 180 models) that contain all 5 features. In order to evaluate the effectiveness of this heuristic, we evaluate all 602 models and find that this heuristic produces the same best model as that produced by an exhaustive search. We select the hybrid model that has the lowest RMSE. The results are described in Section 9.

Step 6: Estimation of Out-of-Sample Error of the Best Models. Once we have selected the best models, we need to estimate their out-of-sample errors. Notice that model selection using 8-fold cross-validation is done using the same training set, and the average RMSE obtained using 100 repetitions of the 8-fold cross-validation process may underestimate the RMSE error for an out-ofsample instance. Therefore, at this stage, we learn the model parameters of the chosen models from the entire training set of 832 cases, and then test it on the test set of 93 cases distinct from the 


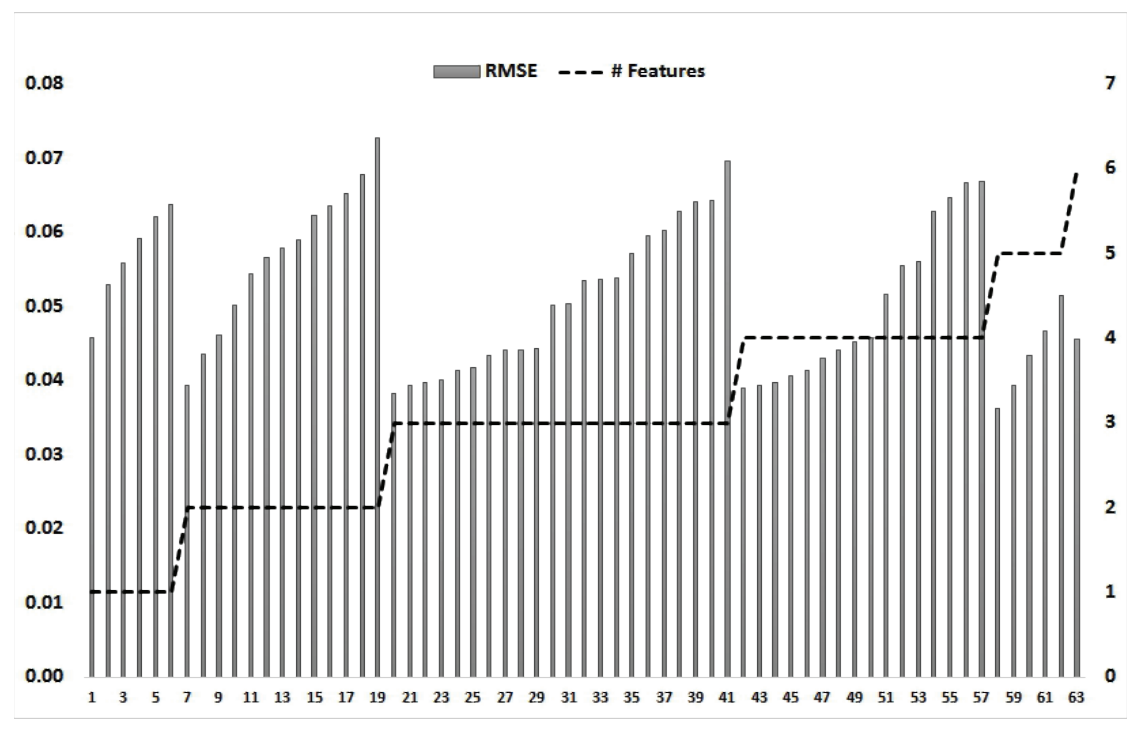

Figure 9: RMSE of LR models with 8-fold CV and 8 bins using the training set

training set. However, this test set error is a point-estimate and we need a measure of variability

(standard error) of the test set error. Since we have only one test set, we use non-parametric bootstrapping technique as proposed by [51] to generate 1000 bootstrap samples, each one of size 93, from the original test set with replacement. From each bootstrap sample, we compute the RMSE and finally compute the standard error and confidence interval. Various bootstrap confidence intervals exist in practice. We report the accelerated and bias-corrected bootstrap confidence intervals $\left(B C_{a}\right)$ proposed by [52]. This approach adjusts for both bias and skewness in the bootstrap distribution and is accurate in a wide variety of settings.

\section{Comparison of Models and Prediction}

In Table 2, we summarize the average RMSE (from 100 repetitions of the 8-fold CV process) of 3 different classifiers on the training set, which is used for model selection. The hybrid model, which has 5 features (GAAP, internal control, bankruptcy, Section 11, and short term drop) emerges as the best model having lowest error on the training set. Using a paired $t$-test at $5 \%$ level of significance, we find that the best hybrid model has significantly smaller RMSE compared to best LR model $(p$-value $=0.04)$ as well as the best NB model $(p$-value $<0.0001)$. Also, the best LR 


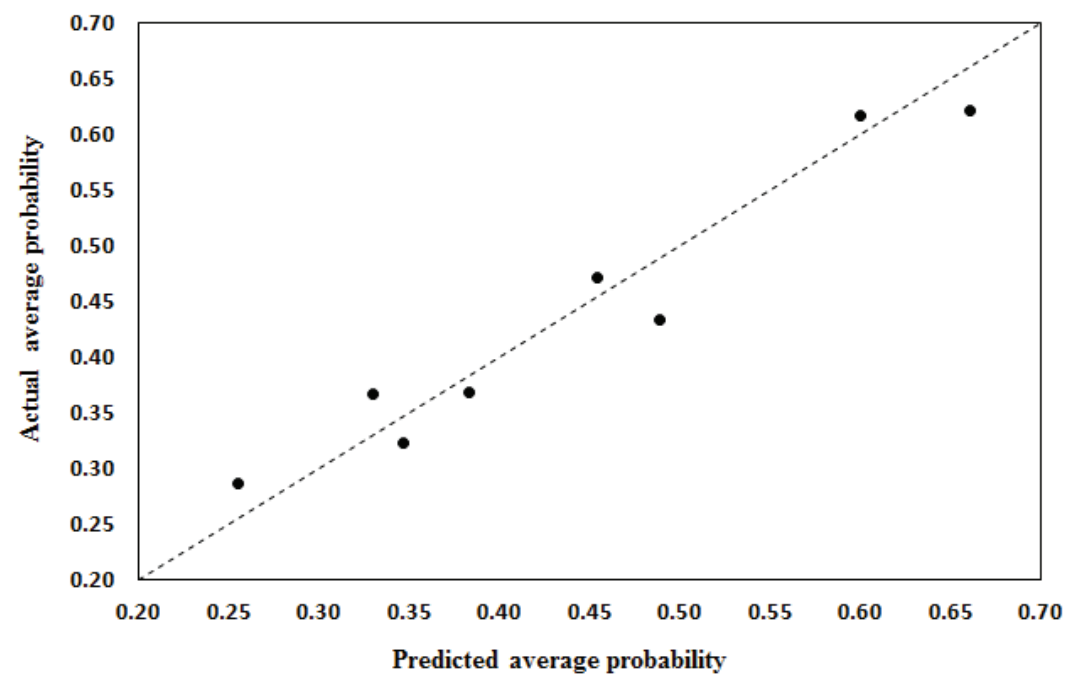

Figure 10: Reliability Diagram of the Best Hybrid Model Using Data in the Training Set

Table 2: Best Models Selected and Their Average RMSE

\begin{tabular}{|lcccc|}
\hline Method & \# features & features & Avg. RMSE & Std. Error \\
\hline Naïve Bayes & 2 & GP, STD & 0.0488 & 0.0012 \\
Logistic Regression & 5 & GP, IC, STD, BR, S11 & 0.0436 & 0.0010 \\
Hybrid & 5 & LR part: GP, IC, BR, S11 & 0.0412 & 0.0011 \\
& & NB part: STD & \\
\hline
\end{tabular}

model is found to be significantly better than the best NB model ( $p$-value is $<0.0002)$ with regard to training set error.

We test the prediction performance of this model on the test set to represent the true error that we might expect on a new dataset. As mentioned above, we resort to bootstrapping (1000 resamples) to estimate the standard error. The test set error of the best hybrid model, along with standard error and confidence interval are given in Table 3. In addition, we use the reliability diagram (see Figure 10) and the bar-chart (see Figure 11) to descriptively evaluate the model performance. It can be seen that the best hybrid model is well-calibrated [53]. 


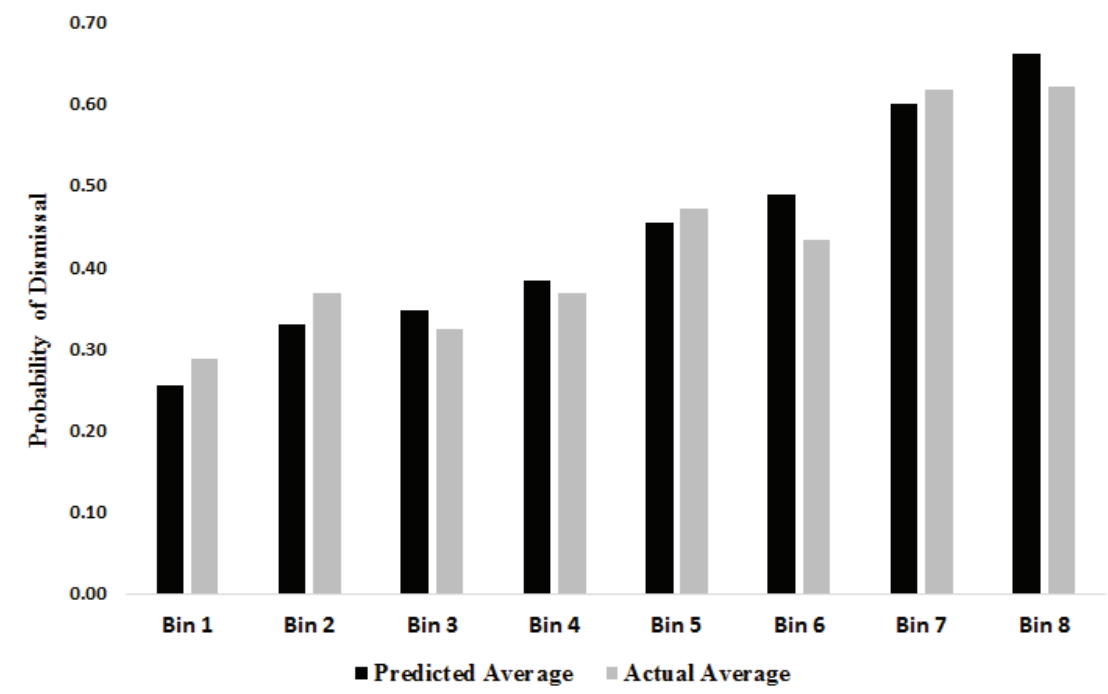

Figure 11: Prediction Performance of the Best Hybrid Model Using Data in the Training Set

Table 3: Test set RMSE

\begin{tabular}{|lccc|}
\hline Method & Avg. RMSE & Bootstrap Std. Error & Bootstrap CI \\
\hline Best Hybrid Model & 0.0930 & 0.0444 & {$[0.0110,0.1843]$} \\
\hline
\end{tabular}

\section{An Example}

To find the parameters of a LR model, we used $g \operatorname{lm}()$ command in stats package in R, which is used for fitting generalized linear models. To find the parameters of a NB model, we used naive.bayes() command in bnlearn package. To find the parameters of a hybrid model, we used the respective packages for the LR and NB part and then combined them using Eq. (9).

Once the best model is chosen, the parameter estimates of the best hybrid model has been obtained using all 925 cases in our dataset. They are as follows: $\beta_{0}=0.6305, \beta_{1}=-0.3412$, $\beta_{2}=-0.2507, \beta_{3}=-1.1506, \beta_{4}=-0.8105$, and the likelihood ratios are $L$ (dismissal, std $=$ $1)=0.6465$, and $L($ dismissal, std $=0)=1.097$. Eq. (10) represents the probability model for predicting the odds of dismissal. We now demonstrate the use of our model using an example, 


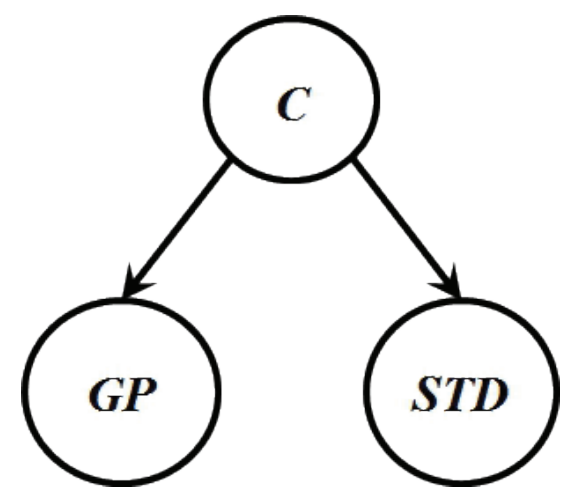

Figure 12: Best Naïve Bayes Model

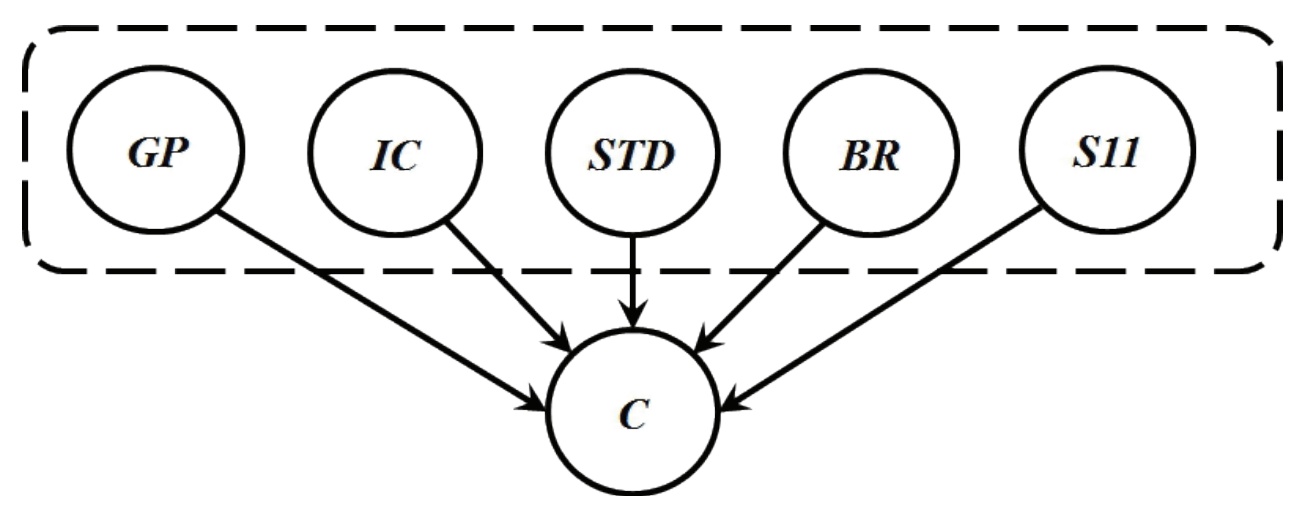

Figure 13: Best Logistic Regression Model

taken from SCAC database, filed in 2013 (outside the sample period).

$$
O(\text { dismissal } \mid g p, i c, b r, s 11, s t d)=e^{\left(\beta_{0}+\beta_{1} g p+\beta_{2} i c+\beta_{3} b r+\beta_{4} s 11\right)} L(\text { dismissal }, \text { std })
$$

Case details: Gail Fialkov, et al. (plaintiff) vs. Microsoft Corporation (defendant); Docket: 13CV-11928; Court: W.D. Washington; Date filed: 08/12/2013. We first identify the values of the

features from the consolidated complaint (See Table 4) and then determine probability of dismissal using Eqs. (11) and (12). As per SCAC database, the case is now dismissed, which is consistent with our prediction. 


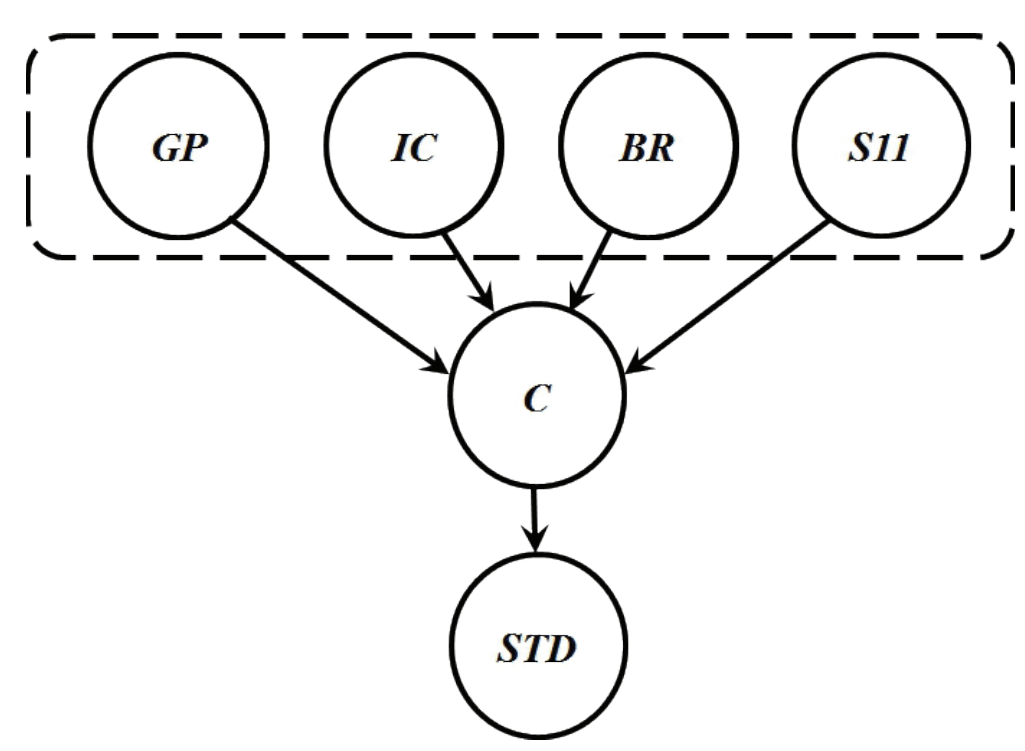

Figure 14: Best Hybrid Model

$$
\begin{array}{r}
O(\text { dismissal } \mid g p=1, i c=1, b r=0, s 11=0, \text { std }=0) \\
=e^{(0.63+(-0.34) \times 1+(-0.25) \times 1+(-1.15) \times 0+(-0.81) \times 0)}(1.09)=1.14
\end{array}
$$

$$
\begin{aligned}
P(\text { dismissal }) & =\frac{O(\text { dismissal })}{O(\text { dismissal })+1} \\
& =0.5327
\end{aligned}
$$

\section{Summary \& Conclusions}

The main contribution of this paper is a model for computing probabilities for dismissal of $10 \mathrm{~b}-5$

securities class-action cases filed in Federal district courts. Using a hybrid model, which combines logistic regression and naïve Bayes, we suggest that only 5 features are significant for predicting the probability of (initial) dismissal. They are GAAP, internal control, bankruptcy, Section 11, and short term drop. Only short term drop, which was discretized into 2 bins with $42.2 \%$ drop as a cutoff value, was used in the NB part. 


\begin{tabular}{|c|c|c|c|}
\hline Predictor & Trie/palse & $\begin{array}{l}\text { Value of the } \\
\text { predictor? }\end{array}$ & Reference from the consolidated complaint \\
\hline $\begin{array}{l}\text { Internal } \\
\text { control }\end{array}$ & Yes & 1 & $\begin{array}{l}\text { The Companv's disclosure controls and its internal controls over } \\
\text { financial reporting were materially deficient and its representations } \\
\text { concerning them during the Class Period were materially false and } \\
\text { misleading; (Clause } 65 \text {, Section g, Page } 21 \text { ) }\end{array}$ \\
\hline Bankruptcy & No & 0 & Not Reported \\
\hline $\operatorname{Sec} 11$ & No & 0 & Not Reported \\
\hline GAAP & No & 1 & $\begin{array}{l}\text { That the Company's financial statements for the quarter ended } \\
\text { March } 31,2013 \text { were materially false and misleading by violating } \\
\text { GAAP and Microsoft's publicly disclosed policy of accounting for } \\
\text { inventories; (Clause } 65 \text {, Section } f \text {, Page } 20 \text { ) }\end{array}$ \\
\hline $\begin{array}{c}\text { Short Term } \\
\text { Drop }\end{array}$ & Yes $(<42.2 \%)$ & $\mathbf{0}$ & $\begin{array}{l}\text { On this news, Microsoft common stock suffered its biggest price } \\
\text { decline in more than four years, plunging } \$ 4.04 \text { per share, or } 11.4 \% \text {, } \\
\text { on very heavy trading volume to close at } \$ 31.40 \text { per share. The } \\
\text { magnitude of the decline in the price of Microsoft's stock } \\
\text { eviscerated about } \$ 34 \text { billion of the Company's market value. } \\
\text { (Clause 13, Page 3) }\end{array}$ \\
\hline
\end{tabular}

Table 4: Determining the value of the features from the consolidated complaint implies that allegation of GAAP violations, lack of internal control, violations of Section 11 of Securities Act 1933, and bankruptcy filing by defendant, all make a class-action case less likely to be dismissed. Our results are in agreement with the key findings in existing literatures. For example, [33], [34] and [13], and all suggest that class-action cases with GAAP violations are less that violations of section 11 makes a case less prone to dismissal. However unlike us, they do not 
find the effect statistically significant.

Our findings also complement the existing literatures, which study the determinants of settlement size. For example, our results suggest that when the defendant firms file for bankruptcy during the class period, the court is less likely to dismiss the case. However, once the case is settled, bankruptcy does not seem to play any role in determining the settlement size [31]. Likewise, [31] shows that presence of institutional lead plaintiff increases the overall settlement size. In this paper, we bring interesting insights by suggesting that the presence of an institutional lead plaintiff, however, does not increase the chance of dismissal.

We do not find insider selling as a significant feature and our result concurs with [32] and [34]. [34] explains that insider selling (a.k.a insider trading) is a commonly pleaded motive for fraud in many complaints and are pleaded more often when stock options is a form of compensation for employees. As a result, the courts are skeptical about this allegation.

However, unlike [32], we do not find restated financials as a significant feature. Generally, restated financials is perceived as direct evidence for accounting violations and makes a suit less likely to be dismissed. In our findings, models that include GAAP violations but not restated financials predict better than those that include both GAAP and restated financials. It is possible that these two features are highly correlated, and including both features results in double counting that lowers the accuracies of the models.

Further, we also observe that the short-term-drop $(S T D)$ in share price within a period of $1-5$ days is an important attribute. This agrees with our belief that STD measures the immediate impact of the security fraud and is a proxy of the financial loss suffered by investors. Hence, we expect the likelihood of dismissal to be lower when share price drop is $>42.2 \%$. In our case, the likelihood ratio for dismissal is 0.6465 when $S T D>42.2 \%$ and 1.097 when $S T D \leq 42.2 \%$, which includes no allegation of short term drop in the consolidated complaint (see Section 10). Thus, an allegation of $S T D>42.2 \%$ favors non-dismissal, which is consistent with our belief.

Finally, our model suggests that an allegation of lack of internal control is an important feature for predicting probability of dismissal. This feature has not been widely studied in the past. However, lack of internal control is being increasingly alleged in security class-actions. On an 
average, $23 \%$ of all class-actions filed during 2011-2015 have alleged internal control weaknesses over financial reporting [35]. Hence, lack of internal control is an important feature for predicting dismissal.

In conclusion, we present a model that can be used to predict probability of dismissal in response to motion to dismiss. The model identifies 5 features that are significant for predicting dismissal, and largely consistent with the existing literature. The proposed hybrid model for computation of probability of dismissal will act a decision support tool for the D\&O insurers in U.S. Since virtually every public corporations in U.S. buy D\&O insurance, there is an increasing interest in predicting the probability of dismissal of class-action cases. Even knowing what features are relevant for predicting probabilities of dismissal is also useful for assessment of underwriting risks for D\&O insurance. Considering the size of class-action settlements and increasing incidence of class-action cases, we believe that an improvement in predictive performance will go a long way.

\section{Acknowledgement}

This project was funded in part by a sponsored research project from a D\&O insurance company (which wishes to remain anonymous) to the Center for Business Analytics Research, School of Business, University of Kansas. We are grateful to the global head of the D\&O company (unnamed for anonymity reasons) for helping us with classifying cases in our dataset as dismissed/not dismissed. Thanks to Suzanna Emelio at University of Kansas for proofreading this manuscript.

\section{References}

[1] M. Mora, O. Gelman, G. Forgionne, F. Cervantes, The implementation of large-scale decision-making support systems: Problems, findings, and challenges, Encyclopedia of Decision Making and Decision Support Technologies $2(2008) 455$.

[2] R. H. Sprague Jr, E. D. Carlson, Building effective decision support systems, Prentice Hall Professional Technical Reference, 1982.

[3] M. Amann, M. Makowski, Effect-focused air quality management, Mathematical Modeling Theory and Applications 9 (2000) 367-398.

[4] S. Strubegger, M. Messner, Model-based decision support in energy planning, International Journal of Global Energy Issues 12 (1-6) (1999) 196-207. 
[5] F. Ghasemzadeh, N. P. Archer, Project portfolio selection through decision support, Decision Support Systems 29 (1) (2000) 73-88.

[16] B. V. Dasarathy, B. V. Sheela, A composite classifier system design: concepts and methodology, Proceedings of

[17] M. Woźniak, M. Graña, E. Corchado, A survey of multiple classifier systems as hybrid systems, Information

Fusion 16 (2014) 3-17.

[18] L. A. Rastrigin, R. H. Erenstein, Method of Collective Recognition, Energoizdat, Moscow, 1981.

[19] R. T. Clemen, Combining forecasts: A review and annotasted bibliography, International journal of forecasting

5 (4) (1989) 559-583.

[20] K. Tumer, J. Ghosh, Analysis of decision boundaries in linearly combined neural classifiers, Pattern Recognition

29 (2) (1996) 341-348.

600

[6] L. Bai, J. D. Cox, R. S. Thomas, Lying and getting caught: An empirical study of the effect of securities class 7] J. C. Coffee, Reforming the securities class action: An essay on deterrence and its implementation, Columbia Law Review 106 (7) (2006) 1534-1586.

[8] T. Eisenberg, G. P. Miller, Attorney fees and expenses in class action settlements: 1993-2008, Journal of Empirical Legal Studies 7 (2) (2010) 248-281.

[9] B. T. Fitzpatrick, An empirical study of class action settlements and their fee awards, Journal of Empirical Legal Studies 7 (4) (2010) 811-846.

[10] T. Baker, S. J. Griffith, Predicting corporate governance risk: Evidence from the directors' and officers' liability insurance market, Chicago Law Review 74 (2007) 487.

[11] T. Baker, S. J. Griffith, How the merits matter: Directors' and officers' insurance and securities settlements,

[12] J. E. Core, On the corporate demand for directors' and officers' insurance, The Journal of Risk and Insurance 64 (1) (1997) 63-87.

[13] B. B. McShane, O. P. Watson, T. Baker, S. J. Griffith, Predicting securities fraud settlements and amounts: A hierarchical Bayesian model of Federal securities class action lawsuits, Journal of Empirical Legal Studies 9 (3) (2012) 482-510.

[14] D. H. Wolpert, The supervised learning no-free-lunch theorems, in: R. Roy, M. Köppen, S. Ovaska, T. Furuhashi, F. Hoffmann (Eds.), Soft Computing and Industry: Recent Applications, Springer, London, 2002, pp. 25-42.

[15] C. K. Chow, Statistical independence and threshold functions, IEEE Transactions on Electronic Computers EC-14 (1) (1965) 66-68.

\section{the IEEE 67 (5) (1979) 708-713.}

[21] R. Raina, Y. Shen, A. McCallum, A. Y. Ng, Classification with hybrid generative/discriminative models, in:

S. Thrun, L. Saul, B. Schölkopf (Eds.), Advances in Neural Information Processing Systems 16, MIT Press, 2004, pp. 545-552. 
[22] Y. D. Rubinstein, T. Hastie, Discriminative vs Informative Learning, in: Proceedings of the Third International Conference on Knowledge Discovery and Data Mining (KDD-97), AAAI Press, 1997, pp. 49-53.

[23] G. Bouchard, B. Triggs, The tradeoff between generative and discriminative classifiers, in: J. Antoch (Ed.), 16th International Symposium on Computational Statistics (COMPSTAT '04), 2004, pp. 721-728.

[24] B. M. Kelm, C. Pal, A. McCallum, Combining generative and discriminative methods for pixel classification with multi-conditional learning, in: 18th International Conference on Pattern Recognition (ICPR'06), Vol. 2, IEEE, 2006, pp. 828-832.

[25] C. M. Bishop, J. Lasserre, et al., Generative or discriminative? Getting the best of both worlds, Bayesian statistics 8 (8) (2007) 3-24.

[26] C. Kang, J. Tian, A hybrid generative/discriminative Bayesian classifier, in: G. Sutcliffe, R. Goebel (Eds.), Proceedings of the 19th International Florida Artificial Intelligence Research Society Conference (FLAIRS-06), 2006, pp. 562-567.

[27] J. H. Xue, D. M. Titterington, Joint discriminative-generative modelling based on statistical tests for classification, Pattern Recognition Letters 31 (9) (2010) 1048-1055.

[28] A. Y. Ng, M. I. Jordan, On discriminative vs. generative classifiers: A comparison of logistic regression and naïve Bayes, in: T. G. Dietterich, S. Becker, Z. Ghahramani (Eds.), Advances in Neural Information Processing Systems 14, MIT Press, 2002, pp. 841-848.

[29] J. H. Xue, D. M. Titterington, On the generative-discriminative tradeoff approach: Interpretation, asymptotic efficiency and classification performance, Computational Statistics \& Data Analysis 54 (2) (2010) 438-451.

[30] J. D. Cox, R. S. Thomas, D. Kiku, Does the plaintiff matter? An empirical analysis of lead plaintiffs in securities class actions, Columbia Law Review 106 (7) (2006) 1587-1640.

[31] J. D. Cox, R. S. Thomas, L. Bai, There are plaintiffs and there are plaintiffs: An empirical analysis of securities class action settlements, Vanderbilt Law Review 61 (2) (2008) 355-386.

[32] M. F. Johnson, K. K. Nelson, A. C. Pritchard, Do the merits matter more? The impact of the Private Securities Litigation Reform Act, Journal of Law, Economics, and Organization 23 (3) (2007) 627-652.

[33] M. Klausner, J. Hegland, When are securities class actions dismissed, when do they settle, and for how much?, Plus Journal 23 (3) (2010) 1-5.

[34] A. C. Pritchard, H. A. Sale, What counts as fraud? An empirical study of motions to dismiss under the Private Securities Litigation Reform Act, Journal of Empirical Legal Studies 2 (1) (2005) 125-149.

[35] A. Aganin, Securities class action filings - 2015 year in review, Research report, Cornerstone Research, Inc. (2016).

[36] R. A. Fisher, The use of multiple measurements in taxonomic problems, Annals of Eugenics 7 (2) (1936) $179-188$.

[37] D. Koller, M. Sahami, Toward optimal feature selection, Technical Report 1996-77, Stanford InfoLab (February 1996).

[38] S. Fu, M. C. Desmarais, Markov blanket based feature selection: A review of past decade, in: Proceedings of 
the World Congress on Engineering, Vol. 1, 2010, pp. 321-328.

[39] S. Hillmer, P. P. Shenoy, Computing the probabilities of closing of 10b-5 securities class action cases, http: //pshenoy.faculty.ku.edu/BUS936/Powerpoint/ShenoySp14.pdf, available Online; January 31, 2014 (2014).

[40] P. Domingos, M. Pazzani, On the optimality of the simple Bayesian classifier under zero-one loss, Machine Learning 29 (2-3) (1997) 103-130.

[41] A. P. Dempster, N. M. Laird, D. B. Rubin, Maximum likelihood from incomplete data via the EM algorithm, Journal of the Royal Statistical Society, Series B 39 (1) (1977) 1-38.

[42] N. L. Zhang, D. Poole, A simple approach to Bayesian network computations, in: Proceedings of the 10th Canadian Conference on Artificial Intelligence, Springer, NY, 1994, pp. 171-178.

[43] M. Scutari, J. B. Denis, Bayesian networks: with examples in R, CRC Press, 2014.

[44] J. Pearl, Probabilistic Reasoning in Intelligent Systems: Networks of Plausible Inference, Morgan Kaufmann, 2014 .

[45] D. Margaritis, Learning Bayesian network model structure from data, Ph.D. thesis, Carnegie Mellon University School of Computer Science, Pittsburgh, PA (2003).

[46] I. Tsamardinos, C. F. Aliferis, A. Statnikov, Algorithms for large scale Markov blanket discovery, in: Proceedings of the Sixteenth International Florida Artificial Intelligence Research Society Conference (FLAIRS-03), 2003, pp. $376-381$.

[47] S. Yaramakala, D. Margaritis, Speculative Markov blanket discovery for optimal feature selection, in: Proceedings of the Fifth IEEE International Conference on Data Mining (ICDM-05), IEEE Computer Society, Washington, DC, 2005, pp. 809-812.

[48] S. Kotsiantis, D. Kanellopoulos, Discretization techniques: A recent survey, GESTS International Transactions on Computer Science and Engineering 32 (1) (2006) 47-58.

[49] U. Fayyad, K. B. Irani, Multi-interval discretization of continuous-valued attributes for classification learning, in: R. Bajcsy (Ed.), Proceedings of the 13th International Joint Conference on Artificial Intelligence, 1993, pp. $1022-1029$.

[50] E. J. Clarke, B. A. Barton, Entropy and MDL discretization of continuous variables for Bayesian belief networks, International Journal of Intelligent Systems 15 (1) (2000) 61-92.

[51] B. Efron, R. Tibshirani, Bootstrap methods for standard errors, confidence intervals, and other measures of statistical accuracy, Statistical Science 1 (1) (1986) 54-75.

[52] B. Efron, Better bootstrap confidence intervals, Journal of the American Statistical Association 82 (397) (1987) $171-185$.

[53] A. Niculescu-Mizil, R. Caruana, Predicting good probabilities with supervised learning, in: Proceedings of the 22nd International Conference on Machine Learning (ICML-05), ACM, 2005, pp. 625-632. 